REGARDS

SUR L'ECONOMIE ALLEMANDE

BULLETIN ECONOMIQUE DU CIRAC

\section{Regards sur l'économie allemande}

Bulletin économique du CIRAC

$90 \mid 2009$

Varia

\title{
Les défis de l'intégration européenne pour la politique énergétique allemande
}

\section{Severin Fischer}

Traducteur : Isabelle Bourgeois

\section{OpenEdition \\ Journals}

Édition électronique

URL : http://journals.openedition.org/rea/3590

DOI : $10.4000 /$ rea.3590

ISBN : 978-2-8218-0876-8

ISSN : 1965-0787

Éditeur

CIRAC

Édition imprimée

Date de publication : 1 mars 2009

Pagination : 31-38

ISSN : 1156-8992

\section{Référence électronique}

Severin Fischer, «Les défis de l'intégration européenne pour la politique énergétique allemande », Regards sur l'économie allemande [En ligne], 90 | mars 2009, mis en ligne le 01 mars 2011, consulté le 10 décembre 2020. URL : http://journals.openedition.org/rea/3590; DOI : https://doi.org/10.4000/rea. 3590 


\title{
Les défis de l'intégration européenne pour la politique énergétique allemande
}

\author{
Severin Fischer
}

La question de la définition d'une politique énergétique figure avec une rare constance en tête de l'agenda communautaire comme des réflexions nationales. La dépendance croissante de l'importation d'énergies fossiles devient de plus en plus préoccupante, comme l'a rappelé avec acuité le conflit russo-ukrainien sur le gaz au début de 2009. Et les voix d'experts comme ceux de l'Intergovernmental Panel on Climate Change (IPCC) avertissant des dangers d'un changement climatique anthropogène se font de plus en plus pressantes. Or ces deux questions étant liées puisque la consommation d'énergie est considérée comme la cause principale du réchauffement climatique pronostiqué, elles forcent les acteurs politiques à agir dans les domaines à la fois de la sécurité de l'approvisionnement énergétique et de la lutte contre le réchauffement climatique. Dans le même temps, ils ont à répondre aux exigences de plus en plus vives des consommateurs (leurs électeurs) en leur garantissant des prix énergétiques les plus bas possibles. Plus que jamais, la politique énergétique se doit aujourd'hui de concilier trois objectifs : la sécurité de l'approvisionnement, le respect de l'environnement et la compétitivité économique.

Cette délicate équation place l'Allemagne devant un défi particulier ; pour trois raisons. L'Allemagne étant le premier importateur d'énergies fossiles dans l'UE, elle est particulièrement exposée aux risques pesant sur son approvisionnement énergétique. Ensuite, étant donné qu'elle est le plus gros producteur de $\mathrm{CO}_{2}$ au sein de I'UE, elle porte une lourde responsabilité dans le domaine de la politique climatique. Enfin, les prix de l'énergie y sont supérieurs à la moyenne européenne, tant pour les ménages que pour l'industrie, ce qui constitue un handicap pour les entreprises allemandes dans le jeu de la concurrence intra-européenne ...

\section{Les lignes d'évolution de la politique énergétique allemande}

Les crises pétrolières de 1973 et 1979/80 ont été déterminantes pour les politiques énergétiques des Etats membres de l'UE en révélant qu'une consommation illimitée de produits pétroliers met en danger prospérité économique et bienêtre de la société, et qu'il convient de contenir ce risque. Mais si l'analyse des problèmes était comparable dans la plupart des Etats, les réponses apportées différaient fondamentalement. La France misa avant tout sur son indépendance énergétique, optant pour l'extension radicale du soutien au nucléaire à la suite du «Plan Messmer». La filière énergétique fut décrétée priorité nationale, et on diversifia l'origine des importations de gaz et de pétrole (Percebois, 2008).

Bien que proches des françaises, dans leurs grandes lignes, les réponses allemandes aux chocs pétroliers se sont traduites par des approches politiques multiples. Comme les autres pays industrialisés, l'Allemagne mit l'accent sur le développement de la filière nucléaire. Mais dans le même temps, la filière charbon, qui avait amorcé son déclin, fut revalorisée, notamment pour des raisons sociales puisque l'extraction de houille et de lignite occupait alors plusieurs centaines de milliers de salariés. Contrairement donc à ce qui fut le cas dans les autres pays où la part de cette ressource dans la consommation primaire d'énergie était en baisse, celle-ci continua et continue toujours de jouer un rôle important dans le mix énergétique outre-Rhin. Dans le même temps également, l'Allemagne développa l'exploitation de ses réserves de pétrole et de gaz naturel dans la mer du

RFA : diversification des choix après les chocs pétroliers 
Nord, chercha à renforcer ses relations avec l'Union soviétique en matière de politique énergétique, lança ses premiers programmes visant à réaliser des économies d'énergie et étendit, quoique timidement encore, sa politique de soutien aux énergies renouvelables (Reiche, 2005).

A cela s'ajoute, à partir des années 1980, la montée en puissance dans la société allemande (mais aussi en Suède ou en Autriche) d'une hostilité croissante au nucléaire qui finit par aboutir à l'abandon de cette ressource sous le premier gouvernement SPD/Verts du chancelier Schröder. La Loi sur la sortie du nucléaire (Atomausstiegsgesetz) entrée en vigueur en 2002 interdit la construction de nouvelles centrales et limite la durée de vie des centrales existantes à 32 ans à partir de leur mise en service. Dans son Traité de coalition conclu en 2005, le gouvernement fédéral d'Angela Merkel proroge explicitement ces dispositions.

En Allemagne, les compétences en matière de politique énergétique sont réparties de plusieurs manières. D’abord, entre Bund et Länder, ces derniers définissant leurs propres politiques dans le cadre de leur politique de développement structurel régional. C'est ainsi que la Bavière, par exemple, s'était hissée au rang de premier site producteur d'électricité nucléaire outre-Rhin (avec $70 \%$ de la production). Ensuite, à l'échelon du Bund, où le ministère fédéral de l'Economie, de la Technologie et de L'Energie (BMWi) formule les grands axes de la politique énergétique de l'Allemagne, mais entre toutefois en concurrence avec le ministère fédéral de l'Environnement, de la Protection de la Nature et de la Sécurité des Réacteurs (BMU). Ce dernier étant responsable de la sécurité des centrales nucléaires, du stockage intermédiaire et définitif des déchets nucléaires, mais aussi de la promotion des énergies renouvelables, ainsi que du système des droits d'émission de gaz à effet de serre, il exerce une influence directe sur les choix de son homologue BMWi. C'est ainsi que le Vert Jürgen Trittin, ministre de l'Environnement sous le premier gouvernement Schröder, avait pu forcer la sortie du nucléaire, l'emportant sur son homologue de l'Economie, le sans parti Werner Müller (Autret, 1999). A la répartition des compétences à cet échelon s'ajoute la question de l'appartenance politique des ministres dans le cadre des coalitions fédérales. Ainsi, sous le gouvernement Merkel s'opposent aujourd'hui d'un côté Sigmar Gabriel, social-démocrate et fervent anti-nucléaire à la tête du BMU et, de l'autre, le chrétien-social KarlTheodor zu Guttenberg qui succède depuis janvier 2009 à Michael Glos à la tête du BMWI. Ces oppositions dues à l'appartenance politique au sein des diverses coalitions fédérales sont bien sûr avivées par les divergences d'approche liées aux champs de compétences des ministères : préoccupations environnementales d'une part, objectifs de compétitivité économique et de sécurité de l'approvisionnement, de l'autre. La politique fédérale en matière d'énergie est donc toujours le fruit d'un savant compromis, à l'instar de ce Programme intégré Energie-Climat (Integriertes Energie- und Klimaprogramm, IEKP) adopté en août 2007 et qui, plus connu sous le nom de « Programme de Meseberg » (d'après le lieu de la réunion) pose les jalons en la matière pour les années à venir.

Or actuellement, si l'interdiction faite aux opérateurs de construire de nouvelles installations fait l'objet d'un large consensus dans tous les partis politiques, il n'en va pas de même de la limitation de la durée de vie des centrales ; cette disposition pourrait être abrogée à l'occasion d'un changement de gouvernement à l'issue des élections au Bundestag de septembre 2009. Et la question de l'avenir du nucléaire pourrait occuper une place centrale dans la campagne électorale.

\section{Une structure énergétique diversifiée et une intensité énergétique efficiente}

Une consommation importante de houille et de lignite

Les renouvelables en hausse dans la production d'électricité
La structure de la consommation d'énergie primaire est très diversifiée outre-Rhin, bien que le pétrole s'y taille encore de loin la part la plus importante $(33,9 \%$ en 2007) du fait qu'il est principalement utilisé dans les transports, la production industrielle et pour le chauffage ; son emploi dans la production d'électricité est résiduelle. La houille $(14,3 \%)$ et le lignite $(11,6 \%)$ ont conservé une place importante dans la consommation d'énergie primaire outre-Rhin. Mais alors que le lignite est extrait dans sa totalité du sol allemand, dans le cas de la houille, depuis plusieurs années, les importations prennent de plus en plus largement le pas sur l'extraction nationale. C'est sur le gaz naturel, bien qu'il représente moins d'un quart $(22,5 \%)$ de l'Energiemix, que se concentrent les débats politiques, du fait de l'origine géographique des importations comme des enjeux liés à sa consommation. Enfin, la part des énergies renouvelables, qui est passée de 5,5\% en 2006 à 6,6 \% en 2007 (en y incluant la biomasse), devrait s'accroître à l'avenir dans les mêmes proportions après l'extension de la Loi sur les énergies renouvelables (Erneuerbare-Energien-Gesetz, EEG) en 2008 et l'annonce de multiples projets d'investissement.

La structure de la production d'électricité présente une évolution bien plus dynamique en comparaison, dans le passé mais aussi dans le futur, du fait des choix politiques effectués : abandon du nucléaire et soutien aux énergies renouvelables. Aujourd'hui (2007), avec près des trois quarts du total, le nucléaire, le lignite et la houille s'y taillent toujours la part du lion ; mais celle-ci diminue depuis 1990. En valeur absolue, malgré la hausse globale de la consommation d'électricité, la part 
de la production d'électricité à partir du lignite et du nucléaire n'a cessé de baisser, tandis que celle provenant de la houille est restée constante dans l'ensemble. La part des énergies renouvelables (surtout l'éolien) comme celle du gaz ont progressivement crû en proportion, passant de moins de $4 \%$ à quelque $11 \%$ pour les premières, de $6,5 \%$ à $11,7 \%$ pour le deuxième. Or en valeur absolue, cette croissance est nettement supérieure étant donné la hausse globale de la consommation d'électricité. La structure de la production d'électricité s'est donc considérablement modifiée outre-Rhin, et cette évolution devrait se poursuivre suite aux effets de l'extension de la loi EEG comme de l'instauration du système européen d'échanges de quotas d'émission de $\mathrm{CO}_{2}$ (Autret, 2005). Dans le domaine de l'électricité plus qu'ailleurs, les entreprises ont en effet besoin de conditions cadres leur offrant une prévisibilité sur le long terme pour l'investissement.

\begin{tabular}{|c|c|c|c|}
\hline \multicolumn{4}{|c|}{ Consommation d'énergie primaire en Allemagne en 2007} \\
\hline Pétrole & $33,9 \%$ & Nucléaire & $11,1 \%$ \\
\hline Gaz naturel & $22,5 \%$ & Hydraulique + éolien & $1,5 \%$ \\
\hline Houille & $14,3 \%$ & Autres & $5,6 \%$ \\
\hline Lignite & $11,6 \%$ & & \\
\hline
\end{tabular}

Source des données : AG Energiebilanzen, 2008

\begin{tabular}{|c|c|c|}
\hline \multicolumn{3}{|c|}{ Evolution de la production d'électricité par source d'énergie en Allemagne } \\
\hline & 1991 & 2007 \\
\hline Total production (milliards de $\mathrm{kW} / \mathrm{h}$ ) & 540,2 & 636,5 \\
\hline \multicolumn{3}{|l|}{ Par source d'énergie : } \\
\hline - Pétrole & $2 \%$ & $1 \%$ \\
\hline - Houille & $27 \%$ & $23 \%$ \\
\hline - Nucléaire & $28 \%$ & $22 \%$ \\
\hline - Gaz naturel & $7 \%$ & $12 \%$ \\
\hline - Lignite & $29 \%$ & $24 \%$ \\
\hline - Hydraulique + éolien & $4 \%$ & $11 \%$ \\
\hline - Autres & $3 \%$ & $7 \%$ \\
\hline Total consommation d'énergie primaire (pétajoules) & 14610 & 13993 \\
\hline
\end{tabular}

Source des données : AG Energiebilanzen, 2008 ; Bundesministerium für Wirtschaft und Technologie, 2008.

En tendance, la hausse de la consommation d'électricité - ou, plutôt, sa stabilisation à niveau constant eu égard à la baisse de la consommation totale d'énergie primaire - devrait se poursuivre au cours des prochaines années. Cette hausse relative est directement liée à l'utilisation croissance d'appareils électriques par les ménages comme à la diffusion attendue de la voiture électrique. La production d'électricité restera donc en tête des préoccupations des milieux politiques.

Pourtant, malgré une consommation globale d'énergie primaire élevée en comparaison européenne, l'économie allemande a réalisé de considérables gains d'efficience en matière d'intensité énergétique. Alors que, dans l'UE 27, l'industrie consomme en moyenne 154 tonnes-équivalent pétrole par point de PIB (150 en France), elle se contente de 141 en Allemagne (Leimbach/Müller, 2008). Et si, en RFA, la consommation par tête (3,99 tonnes-équivalent pétrole) est supérieure à la moyenne de l'UE $(3,36)$, elle est inférieure à celle enregistrée en France $(4,41)$.

\section{Le gaz, un important facteur de risque}

L'interruption des livraisons de gaz russe via l'Ukraine au début de 2009 et son impact sur l'économie comme la conscience du risque qu'elle a nourri dans l'opinion ont placé la problématique du gaz naturel au centre des préoccupations, surtout sous l'angle de la structure des importations. Certes, les fournisseurs russes n'ont cessé de rassurer les ménages comme les électriciens sur la sécurité de l'approvisionnement tout au long des trois semaines qu'a duré l'interruption, tout en multipliant les voies de contournement en Europe du sud-est pour remplir leurs contrats de livraison. Mais les perspectives sur le long terme sont moins rassurantes.

Car l'Allemagne est fortement tributaire du gaz naturel, aussi bien en matière de chauffage que de production d'électricité. Ainsi, 18,4 millions de logements étaient
La consommation d'électricité se stabilisera à haut niveau

Forte efficience énergétique de l'industrie

Plus de la moitié des logements est chauffée au gaz 
Le gaz, stratégique pour la production d'électricité en pointe

L'Allemagne importe $85 \%$ de son gaz, et son $1^{\text {er }}$ fournisseur est la Russie

Nouer d'excellentes relations commerciales bilatérales...

... ne suffit plus à garantir la sécurité de l'approvisionnement dans le contexte européen chauffés au gaz en 2007 , soit $48,3 \%$ du parc. Dans le neuf, la proportion est nettement supérieure et s'accroît depuis plusieurs années : les autorisations de permis de construire révèlent que $66 \%$ des nouveaux logements sont chauffés au gaz (AGEB, 2008). Dans le domaine du chauffage, la dépendance est donc très forte.

Elle l'est plus encore en matière de production d'électricité, sous l'effet de la politique menée en Allemagne comme dans l'UE contre le changement climatique. D'abord, l'introduction de quotas d'émission de gaz à effet de serre rend, à moyen terme, le gaz plus attractif que le lignite ou la houille, plus riches en $\mathrm{CO}_{2}$, ce qui se traduira par une hausse de la consommation de gaz naturel. Ensuite, l'augmentation décidée de la part des énergies renouvelables dans la production d'électricité, et en priorité celle de l'éolien, exige en corollaire l'extension des capacités en pointe pour pallier les fluctuations de la production liées aux variations météorologiques. Or les centrales au charbon ou nucléaires étant destinées pour l'essentiel à la production en base, seules les centrales au gaz sont à même d'apporter la réactivité exigée en pointe. Enfin, le choix national de la sortie du nucléaire induira à terme une situation de pénurie dans la structure de l'approvisionnement en électricité, à laquelle il ne pourra être remédié que par un recours accru à la ressource gaz. Car si, théoriquement, deux options se présentent, les choix d'investissement privilégieront vraisemblablement les centrales au gaz, plus rentables en termes de coûts et de politique climatique.

Or les ressources allemandes ou européennes en gaz naturel ne suffiront pas pour alimenter un parc de centrales en pleine extension. Actuellement, $15 \%$ seulement du gaz consommé outre-Rhin provient de gisements allemands; tout le reste est importé. Avec un bon tiers du total, le premier fournisseur est la Russie ; son importance devrait croître avec le recul de l'exploitation des gisements de la mer du Nord. Dans cette perspective, les conflits récurrents entre la Russie et ses voisins, les incertitudes quant à la sécurité des infrastructures russes, ainsi que la faiblesse notoire des investissements de la société nationale Gazprom dans la prospection de nouveaux gisements soulèvent un certain nombre de questions.

\begin{tabular}{|lrlr|}
\hline \multicolumn{5}{c|}{ Allemagne. Structure de l'approvisionnement en gaz par origine géographique en 2007} \\
Extraction en RFA & $15 \%$ & Importation des Pays Bas & $18 \%$ \\
Importation de Russie & $37 \%$ & Importation de Grande-Bretagne/Danemark & $4 \%$ \\
Importation de Norvège & $27 \%$ & & \\
\hline
\end{tabular}

Source des données : AG Energiebilanzen, 2008.

Les énergéticiens allemands entretiennent depuis toujours de bonnes relations avec la Russie et les institutions en charge du commerce gazier. Jamais, dans l'histoire de la RFA, les livraisons vers l'Allemagne n'ayant été interrompues sauf par intermittence, et jamais par choix politique délibéré, pouvoirs publics et énergéticiens ne cessent de rappeler à l'opinion la sécurité de l'approvisionnement en gaz russe, soulignant l'excellente qualité des relations germano-russes dans le domaine de l'énergie que prouve le projet de construction du pipeline qui relie Vyborg en Russie à Greifswald en Allemagne via la mer Baltique (« North Stream »).

Mais la sécurité de l'approvisionnement dépasse depuis longtemps le cadre national (et des relations bilatérales qui s'y nouent) et ne peut d'ores et déjà être garantie qu'à l'échelon européen du fait de l'intégration en cours du marché énergétique communautaire dans le fil de sa libéralisation. Même si l'Allemagne parvenait à garantir son approvisionnement en gaz à moyen terme, la dimension européenne des questions énergétiques appelle d'autres réponses.

\section{L'européanisation de la politique énergétique allemande}

Bien que la question énergétique ait joué un rôle central dans le processus d'intégration communautaire, du Traité CECA au Traité Euratom, il aura fallu attendre les Sommets de mars 2007 puis de décembre 2008 du Conseil européen pour que se dégage dans l'UE une politique plus concrète en la matière (Geden/Fischer 2008). Les Etats membres se sont alors mis d'accord sur un certain nombre d'objectifs conjoints : la hausse progressive jusqu'à $20 \%$ de la part des énergies renouvelables dans la consommation d'énergie primaire, la réduction de 
$20 \%$ par rapport à 1990 des émissions de gaz à effet de serre, ou l'accroissement de $20 \%$ de l'efficience énergétique par rapport aux prévisions 2020.

Mais les instances communautaires n'ont de compétences qu'en matière d'environnement et de marché intérieur ; c'est donc via celles-ci que s'est esquissé au fil des ans une sorte de cadre réglementaire 'd'emprunt' pour le secteur énergétique dans l'UE suivant trois axes : libéralisation des marchés de l'électricité et du gaz, promotion des énergies renouvelables et prémisses d'une politique climatique. Institutionnellement, I'UE ne peut donc pas influer sur les choix effectués par les Etats membres en matière de mix énergétique. Or ces choix-là sont déterminants pour la réalisation des trois objectifs supérieurs que sont la sécurisation de l'approvisionnement, le respect de l'environnement et la compétitivité économique. Comment donc l'Allemagne cherche-t-elle à concilier ses impératifs propres avec l'européanisation croissante de la politique énergétique?

\section{Une sécurité de l'approvisionnement à construire à l'échelle de l'UE}

Contrairement à ce qu'affirme le discours officiel, l'approvisionnement de l'Allemagne en gaz naturel est loin d'être garanti, affirme la fédération du commerce extérieur de pétrole et d'énergie, l'AFM+E (Außenhandelsverband für Mineralöl und Energie), rappelant qu'aucun débat public n'aborde cette problématique en profondeur et que les responsables "se contentent de rappeler que, jusqu'ici, l'approvisionnement a toujours fonctionné sans encombre " (AFM+E, 2008). L'Allemagne dispose certes de capacités de stockage permettant d'assurer 40 jours de consommation, mais elles ne sont pas destinées à la prévention de crises ; elles servent les seuls intérêts commerciaux des opérateurs. De plus, nul ne connaît la quantité de gaz naturel effectivement stockée. Quant aux contrats de livraison, ils échappent au contrôle de l'Etat. Le fait qu'en Allemagne la mission de sécuriser l'approvisionnement relève des opérateurs eux-mêmes et de moins en moins de l'Etat résulte de l'adoption en 1998 de la loi Energiewirtschaftsgesetz (EnWG) dont l'objectif était la libéralisation du secteur. Or la concurrence accrue dans le fil de cette libéralisation a contraint les opérateurs à donner priorité à l'efficience de leur gestion au détriment de la prévention. Autrement dit, la 'régulation' du marché gazier allemand repose sur la seule confiance que l'Etat porte aux opérateurs qui ont prouvé leur efficience lors des crises passées.

Le conflit russo-ukrainien sur la livraison de gaz a rappelé par ailleurs l'absence de réglementation de la sécurité de l'approvisionnement à l'échelon communautaire. L'UE n'a en effet aucune compétence dans ce domaine et est donc tributaire de la négociation d'un consensus par les ministres compétents des Etats membres. Alors que, pour le pétrole, a été adoptée il y a quelques années l'obligation de constituer des réserves en prévision de crises (équivalentes à 90 jours de consommation), il n'existe aucune disposition comparable pour le gaz. Certes, une directive de 2004 fait prescription aux Etats membres de développer un système de gestion de crise en cas de rupture d'approvisionnement, mais sans plus de précisions. On comprend dès lors l'absence de coordination institutionnelle des réactions dans l'UE lors de l'interruption des livraisons de gaz russe ; la gestion de la crise a été exclusivement le fait d'un système d'entraide établi sur la base du volontariat par les opérateurs eux-mêmes.

La sécurité de l'approvisionnement implique par ailleurs l'existence d'infrastructures adéquates. L'UE peut certes, à l'aide du programme "Réseaux transeuropéens » pour l'énergie (TEN-E), définir des priorités pour le développement futur des réseaux, mais elle ne dispose pas des moyens budgétaires requis pour inciter les Etats membres à les mettre en œuvre. Un exemple patent de cette situation est l'interminable débat sur le projet de construction du pipeline Nabucco destiné à transporter vers le territoire européen du gaz naturel en provenance d'Asie centrale, de la région de la mer Caspienne et, lorsque la situation politique le permettra, d'Iran ; le tracé de ce pipeline contourne la Russie. Comme, indépendamment de la question du bien-fondé de ce projet, se pose également celle de son financement, le pipeline Nabucco devrait rester longtemps encore un vœu pieu.

Actuellement, pour remédier à l'insuffisance des investissements dans le domaine des infrastructures, le gouvernement fédéral prépare un projet de loi sur le
L'UE n'a pas de compétences directes en matière d'énergie

Allemagne : une auto-régulation inadaptée à la gestion de crise

Pour le gaz, pas d'obligation de constituer des réserves

Des infrastructures transeuropéennes insuffisantes

Un projet de loi en préparation outre-Rhin 
Comment réduire les émissions de $\mathrm{CO}_{2}$ tout en limitant les risques de dépendance?

Le stockage du $\mathrm{CO}_{2}$, la panacée?

L'Allemagne, championne des énergies renouvelables transport du gaz et de l'électricité (Energieleitungsgesetz) visant à réduire les barrières bureaucratiques à leur construction et à améliorer les interconnexions au sein du réseau. Or les mesures nationales ne suffisant plus à garantir la sécurité de l'approvisionnement, il conviendrait désormais d'adopter une réglementation communautaire. Celle-ci permettrait d'assurer une plus grande transparence quant à l'état des réserves de gaz grâce à un contrôle étatique accru; et elle permettrait aussi de supprimer les effets d'aubaine. II conviendrait de même d'adopter un programme conséquent d'investissement dans la création de nouveaux réseaux d'infrastructures, principalement transfrontaliers. Or, invoquant des réserves juridiques sur le financement d'une telle entreprise, le gouvernement fédéral s'oppose à la mise en œuvre d'un projet en ce sens développé par la Commission en janvier 2009 (COM(2009) 35 final).

\section{Politique climatique : la quadrature du cercle ?}

L'entrée en vigueur, en 2005, du système européen d'échanges de droits d'émission de $\mathrm{CO}_{2}$ a créé un cadre réglementaire (en application de la Directive 2003/87/CE) s'appliquant à tous les Etats membres. Si le gouvernement fédéral souscrit à une telle évolution, il n'en a pas moins, dans le contexte des négociations sur le paquet Energie-Climat en décembre 2008, plaidé pour l'adoption d'un régime d'exception applicable à certains secteurs industriels afin de préserver leur compétitivité.

La politique climatique européenne n'est pas sans effets non plus sur la structure allemande d'approvisionnement en énergie, tout particulièrement en électricité. Car l'entrée en vigueur de la nouvelle Directive sur les quotas d'émission de $\mathrm{CO}_{2}$ (votée par le Parlement européen le 17-12-2008, mais non encore publiée au JOCE) réduira la rentabilité d'une production d'électricité largement tributaire du charbon et du lignite. Fortement productrices de $\mathrm{CO}_{2}$, ces deux ressources devraient considérablement voir augmenter leur coût, ce qui se traduira à moyen terme par un recul de leur part dans l'Energiemix allemand au profit du gaz naturel et accroîtra encore la part des ressources importées. Certes, le recours accru au gaz assurera une plus grande flexibilité à la production d'électricité, ce qui aura un effet bénéfique sur la compétitivité allemande dans le contexte européen, mais renforcera aussi sa dépendance énergétique, ce qui peut se révéler problématique pour l'ensemble de l'UE. Or comme l'Allemagne avait opté pour l'abandon du nucléaire, il conviendra également de remplacer cette ressource dans le mix énergétique. La seule voie pour faire face à une dépendance démultipliée sera une stratégie forcée d'économies d'énergie et d'une quête systématique de gains d'efficience dans la consommation d'électricité.

C'est ainsi que le ministère fédéral de l'Environnement a présenté en janvier 2009 un «Roadmap Energiepolitik 2020 » esquissant en dix points une politique énergétique future dans le contexte européen. II prône, entre autres, la mise en œuvre des nouvelles technologies de capture et de stockage de $\mathrm{CO}_{2}$ (CCS) afin de concilier une part de $40 \%$ de charbon et lignite dans la production d'électricité avec les normes environnementales tout en réduisant ainsi à $8 \%$ la part du nucléaire dans l'Energiemix allemand. De son côté, le groupe d'experts Projektgruppe Energiepolitisches Programm (PEPP) du ministère fédéral de l'Economie a présenté, le 5 février, ses propres orientations sur le long terme, dont la mesure clef est le projet d'une "Constitution sur l'énergie » (Energieverfassung), autant de conditions cadre pour ménager la compétitivité du site Allemagne.

Le second axe suivi par l'UE est le développement des énergies renouvelables. L'Allemagne a joué un rôle de promoteur dans l'adoption, en décembre 2008, de la Directive sur les énergies renouvelables (votée par le Parlement européen le 17-12-2008, mais non encore publiée au JOCE), prônant le développement forcé des ressources régénératives. Ce faisant, elle défend sa compétitivité dans un domaine créateur d'emplois (quelque 170000 en 2008, selon la fédération Bundesverband Erneuerbare Energien) et où elle est devenue un des sites les plus attractifs de l'UE pour tous les secteurs industriels impliqués dans l'éolien ou les centrales photovoltaïques depuis que le premier gouvernement Schröder avait mis en œuvre une politique active de soutien à celles-ci par la loi EEG de 1998. 
Dans le cadre de la Directive sur les énergies renouvelables, l'Allemagne s'est engagée à porter à $18 \%$ d'ici 2020 la part de ces dernières dans sa consommation globale d'énergie. Mais le choix des options (biocarburants par exemple) et des secteurs concernés (transports, chauffage et/ou production d'électricité) est laissé à l'appréciation des Etats membres. En Allemagne, du fait de l'abandon du nucléaire, on s'attend ainsi à une forte expansion de l'éolien offshore. Or celleci appelle en retour d'importants investissements dans le développement des infrastructures, principalement sur l'axe nord-sud, afin d'acheminer vers le sud du pays, gros consommateur, l'électricité produite par les installations éoliennes en mer du Nord et en mer Baltique. Et pour compenser la production de pointe qui est le propre des éoliennes, il faudra recourir de manière accrue à la ressource gaz (production en base), ce qui posera avec une acuité nouvelle la question de la sécurité de l'approvisionnement.

La troisième priorité de la politique énergétique européenne est, depuis 2006, le développement de l'efficience énergétique, axe confirmé en novembre 2008 par le rapport de la Commission sur la Deuxième analyse stratégique de la politique énergétique (COM(2008) 781 final), puisqu'il permet de réduire la dépendance, de diminuer les émissions de gaz à effet de serre et d'abaisser les coûts pour le consommateur final. Mais cet objectif, qui fait l'unanimité au plan politique, soulève d'importants problèmes technologiques et financiers; dans la mesure où les investissements dans ces technologies alternatives ne peuvent être amortis que sur le long terme, les sources de financement sont par nature limitées. Néanmoins, l'Allemagne s'efforce de réduire durablement sa consommation d'énergie, notamment dans le cadre de son «Initiative pour l'efficience énergétique » (Initiative EnergieEffizienz) lancée en 2002 et qui associe l'Agence allemande de l'Energie (Deutsche Energie-Agentur, DENA) créée dans ce but en 2000, le ministère fédéral de l'Economie et les entreprises du secteur avec pour principal objectif d'informer l'ensemble des acteurs sur les économies réalisables. II n'en reste pas moins qu'une telle politique ne pourra porter ses fruits qu'à long terme.

\section{Un marché de l'énergie sans véritable concurrence}

La politique énergétique développée par la Commission européenne visait dans un premier temps la constitution d'un marché communautaire de l'électricité et du gaz naturel. Or très vite, cette approche s'est heurtée à la diversité non seulement de la structuration des marchés énergétiques nationaux, mais aussi de l'image dont jouit dans l'opinion de chaque Etat membre la filière énergétique. En France, le monopole de l'opérateur historique EdF commence seulement à être levé avec la fusion GdF/Suez et l'ouverture du marché du gaz et de l'électricité à de nouveaux acteurs privés. En Allemagne, où le marché présente une structure régionalisée, celui-ci a été totalement libéralisé en 1998. L'absence de régulation s'est traduite par un mouvement de concentration, les opérateurs régionaux (au statut de collectivités de droit public) fusionnant pour constituer les quatre grands groupes qui, aujourd'hui, dominent $80 \%$ du marché de l'électricité (Kemfert, 2006). Mais ils souffrent d'une mauvaise image dans l'opinion du fait des prix élevés et peu transparents qu'ils pratiquent, de pratiques d'ententes portées devant l'Office fédéral des cartels, comme de la structure peu économe en $\mathrm{CO}_{2}$ de leur production. Quant au marché du gaz, il est contrôlé à hauteur de 80 \% par trois opérateurs seulement. En effet, le marché allemand de l'énergie n'est régulé que depuis 2005, après que la montée en puissance de la concurrence au sein du marché communautaire a rendu nécessaire l'adoption d'une régulation sectorielle spécifique. Après une réforme de la Loi sur le secteur de l'énergie (Energiewirtschaftsgesetz, EnWG) intervenue la même année, les pouvoirs de l'ancienne autorité de régulation des télécommunications et des services postaux ont alors été élargis au secteur de l'énergie (et des chemins de fer), et l'autorité compétente rebaptisée «Agence fédérale des réseaux 》 (Bundesnetzagentur; voir REA63/03).

Or la position dominante de ces quelques opérateurs sur les marchés régionaux mène, selon la Commission (SEC(2007) 1179), à des distorsions de concurrence sur le marché énergétique allemand, dont pâtissent aussi bien les consommateurs que les nouveaux entrants. Pour y remédier, dans le projet de Troisième Paquet relatif aux marchés de l'électricité et du gaz de l'UE, elle préconise la séparation
Promouvoir l'éolien offshore requiert d'autres infrastructures

Réduire la consommation, un objectif complexe et de long terme

Lente montée en puissance de la nécessité d'une régulation

L'Allemagne refuse elle aussi l'unbundling 
L'intégration croissante du marché européen plaide pour une régulation au niveau de I'UE patrimoniale des différentes activités des opérateurs (unbundling) : activités de réseaux, extraction, production, importation et distribution. Or ces préconisations se heurtent à une forte résistance des pouvoirs publics français et allemands. Ces derniers redoutent qu'un tel " démantèlement » nuise à leurs « champions » que sont E.on ou RWE. Plus important encore, en multipliant les échelons politiques et les acteurs concernés, l'unbundling remettrait en question le délicat équilibre actuel en matière de sécurité de l'approvisionnement.

Même si l'avenir de ce projet de Troisième Paquet est incertain, on voit d'ores-etdéjà s'esquisser une évolution des marchés énergétiques des Etats membres vers la préfiguration d'un marché énergétique communautaire. Dans cette perspective, le projet d'instauration d'une Agence européenne de coopération des régulateurs nationaux (COM(2007) 530 final) est un premier pas vers la constitution d'une instance communautaire de régulation. L'interconnexion croissante des réseaux au sein de l'UE comme le développement du marché intra-européen des droits d'émission plaident en tout cas pour l'adoption prochaine d'une régulation communautaire du marché de l'énergie.

LES POLITIQUES ÉNERGÉTIQUES NATIONALES sont aujourd'hui largement déterminées par «Bruxelles». Car bien que l'échelon européen n'influence pas directement la structure de l'approvisionnement énergétique des Etats membres, il n'en réduit pas moins la marge de manœuvre des gouvernements nationaux en matière de droit de la concurrence ou de politique climatique. Mais que les orientations politiques émanent de l'échelon national ou communautaire, les décideurs ont la responsabilité d'évaluer les conséquences futures de leurs choix - a fortiori dans un domaine comme l'énergie, où l'investissement s'effectue sur le long terme. Priorité devrait donc être donnée à l'investissement dans la recherche sur les technologies liées à l'énergie, à une promotion plus déterminée des économies d'énergie comme des gains d'efficience. Si ces objectifs globaux sont consensuels outre-Rhin, les approches pour leur réalisation diffèrent du fait de la répartition institutionnelle des compétences de politique énergétique au sein du gouvernement fédéral, scindées en deux logiques : préservation de la compétitivité économique versus préservation de l'environnement. La période de pré-campagne pour les élections au Bundestag, qui s'amorce les rend actuellement d'autant plus inconciliables qu'elles confrontent invariablement toutes les parties à l'épineuse question d'un Energiemix déséquilibré par un choix politique du passé : l'abandon du nucléaire.

Traduction : I. Bourgeois

\section{Indications bibliographiques}

- AG Energiebilanzen (AGEB), Energieverbrauch in Deutschland im Jahr 2007, Berlin, 2008

- Aubenhandelsverband für Mineralöl und Energie (AFM+E), Gasbevorratung in Deutschland. Sicherheitsrisiko ? Ein Diskussionsbeitrag, Hambourg, 2008

- AUtret F., "La politique énergétique sous la présidence allemande de l'UE », Regards sur l'économie allemande, $\mathrm{n}^{\circ} 82 / 07$, « Une politique énergétique entre compétitivité et environnement », Regards sur l'économie allemande, $n^{\circ} 72 / 05$, et « L'avenir de la filière nucléaire civile », Regards sur l'économie allemande, $\mathrm{n}^{\circ}$ 41/1999

- Bundesanstalt für Geowissenschaften Und Rohstoffe, Reserven, Ressourcen und Verfügbarkeit von Energierohstoffen 2007, décembre 2007 (www.bgr.bund.de)

- Eckpunkte für ein integriertes Energie- und Klimaprogramm („Programme de Meseberg“), août 2007 (www.bmu.bund.de/files/pdfs/allgemein/application/pdf/klimapaket_aug2007.pdf)

- Geden O., Fischer S., Die Energie- und Klimapolitik der Europäischen Union. Bestandsaufnahme und Perspektiven, Baden-Baden, 2008

- HypoVereinsbanK, Power für Deutschland - Energieversorgung im 21. Jahrhundert, juillet 2008 (www.hvb.de)

- Kemfert C., « Die deutsche Energiepolitik braucht eine Trendwende - Ein 10-Punkte-Plan für eine nachhaltige Energieversorgung ", Discussion Paper n618, DIW, Berlin, 2006

- Leimbach B., MüLleR F., «European Energy Policy : Balancing national interests and the need for policy change. The current European energy dialogue ", Documentation 1/2008, FriedrichEbert-Stiftung, Berlin, 2008

- Percebois J., «Französische Energiepolitik: von der Unabhängigkeit zur Interdependenz », DGAP-Analyse $n^{\circ}$ 9, décembre 2008, Deutsche Gesellschaft für Auswärtige Politik, Berlin, 2008

- REICHE D., Grundlagen der Energiepolitik, Francfort/Main, 2005

- www.bmu.de

- www.bmwi.de. 